\title{
Cobertura de vacinação anti-influenza em idosos, Minas Gerais, Brasil
}

\author{
Coverage of anti-influenza vaccination in the elderly, Minas Gerais, Brazil \\ Cobertura de la vacunación contra la influenza en ancianos, Minas Gerais, Brasil
}

Recebido: 19/04/2021 | Revisado: 03/05/2021 | Aceito: 06/05/2021 | Publicado: 20/05/2021

Pedro Victor de Carvalho Silva ORCID: https://orcid.org/0000-0003-4805-6794 Universidade Federal de São João del-Rei, Brasil

E-mail: upedrovictor1997@gmail.com

Luciana Helena da Silva Nicoli ORCID: https://orcid.org/0000-0003-1495-8255 Universidade Federal de São João del-Rei, Brasil

E-mail: luciananicoli@hotmail.com

Stênio Henrique Oliveira ORCID: https://orcid.org/0000-0002-9169-2262 Universidade Federal de São João del-Rei, Brasil

E-mail: stenioenf@aluno.ufsj.edu.br

Laís Oliveira de Moraes Tavares ORCID: https://orcid.org/0000-0002-6603-775X Universidade Federal de São João del-Rei, Brasil E-mail: laisoliveiramt@gmail.com

Eliete Albano de Azevedo Guimarães ORCID: https://orcid.org/0000-0001-9236-8643 Universidade Federal de São João del-Rei, Brasil E-mail: elietealbano@ufsj.edu.br

Valéria Conceição de Oliveira

ORCID: https://orcid.org/0000-0003-2606-9754 Universidade Federal de São João del-Rei, Brasil E-mail: valeriaoliveira@ufsj.edu.br

\begin{abstract}
Resumo
A influenza é uma infecção viral aguda, imunoprevenível, de transmissão causada por meio das secreções respiratórias de pessoa contaminada pelo vírus influenza. Após a introdução da vacina de Influenza no calendário de vacinação do idoso foi possível notar a redução dos casos de pneumonias e de óbitos por complicações decorrentes da influenza. Tendo em vista a importância da vacinação para o idoso, este estudo objetivou analisar a cobertura vacinal anti-influenza em idosos em Minas Gerais (MG). Método: Trata-se de um estudo transversal, utilizando os dados da campanha de vacinação de 2017. Os dados foram coletados das bases do Sistema de Informação do Programa Nacional de Imunização - SIPNI, Sistema de Informação da Atenção Básica - SISAB e Instituto Brasileiro de Geografia e Estatística - IBGE. Resultados: A cobertura vacinal anti-influenza na Região Oeste foi de 95,6\%. Entre os 53 municípios desta região, a maioria $(84,9 \%$ ) alcançou a meta de cobertura vacinal de influenza de $90 \%$, conforme preconizado. O porte populacional e a cobertura de equipe de saúde da família dos municípios não estiveram associadas à cobertura vacinal, apresentando um p valor de 0,697 e 0,949, respectivamente. Conclusão: O estudo demonstrou alta cobertura vacinal em idosos em Região Oeste de MG e essa cobertura não foi influenciada pela presença de EFS e porte populacional dos municípios. Palavras-chave: Vacinas; Vírus influenza A; Idoso; Imunização; Cobertura vacinal.
\end{abstract}

\begin{abstract}
Influenza is an acute viral infection, immunopreventable, transmitted through the respiratory secretions of a person infected by the influenza virus. After the introduction of the Influenza vaccine in the vaccination calendar of the elderly, it was possible to notice a reduction in cases of pneumonia and deaths due to complications resulting from influenza. In view of the importance of vaccination for the elderly, this study aimed to analyze the anti-influenza vaccine coverage in the elderly in Minas Gerais (MG). Method: This is a cross-sectional study, using data from the 2017 vaccination campaign. Data were collected from the bases of the Information System of the National Immunization Program SIPNI, Primary Care Information System - SISAB and Instituto Brasileiro de Geography and Statistics - IBGE. Results: The anti-influenza vaccine coverage in the West Region was 95.6\%. Among the 53 municipalities in this region, the majority $(84.9 \%)$ reached the goal of $90 \%$ influenza vaccination coverage, as recommended. Population size and family health team coverage in the municipalities were not associated with vaccination coverage, with a p-value of 0.697 and 0.949 , respectively. Conclusion: The study demonstrated high vaccination coverage in the elderly in the Western Region of MG and this coverage was not influenced by the presence of SAI and the population size of the municipalities.
\end{abstract}


Research, Society and Development, v. 10, n. 6, e3610615222, 2021

(CC BY 4.0) | ISSN 2525-3409 | DOI: http://dx.doi.org/10.33448/rsd-v10i6.15222

Keywords: Vaccines; Influenza virus A; Elderly; Immunization; Vaccination coverage.

\begin{abstract}
Resumen
La influenza es una infección viral aguda, prevenible, que se transmite a través de las secreciones respiratorias de una persona infectada por el virus de la influenza. Después de la introducción de la vacuna contra la influenza en el calendario de vacunación de los ancianos, se pudo notar una reducción en los casos de neumonía y muertes por complicaciones derivadas de la influenza. Dada la importancia de la vacunación de los ancianos, este estudio tuvo como objetivo analizar la cobertura de la vacuna antigripal en los ancianos de Minas Gerais (MG). Método: Se trata de un estudio transversal, utilizando datos de la campaña de vacunación de 2017. Los datos fueron recolectados de las bases del Sistema de Información del Programa Nacional de Inmunizaciones - SIPNI, Sistema de Información de Atención Primaria - SISAB e Instituto Brasileiro de Geografía y Estadística - IBGE. Resultados: La cobertura de la vacuna antigripal en la Región Oeste fue del 95,6\%. Entre los 53 municipios de esta región, la mayoría (84,9\%) alcanzó la meta del $90 \%$ de cobertura de vacunación contra la influenza, como se recomendó. El tamaño de la población y la cobertura del equipo de salud familiar en los municipios no se asociaron con la cobertura de vacunación, con un valor de $\mathrm{p}$ de 0,697 y 0,949, respectivamente. Conclusión: El estudio demostró alta cobertura de vacunación en ancianos de la Región Occidental de MG y esta cobertura no fue influenciada por la presencia de EFS y el tamaño poblacional de los municipios.
\end{abstract}

Palabras clave: Vacunas; Virus de la influenza A; Ancianos; Inmunización; Cobertura de vacunación.

\title{
1. Introdução
}

A influenza é uma infecção viral aguda de transmissão causada por meio das secreções respiratórias de pessoa contaminada pelo vírus influenza. Tem elevada transmissibilidade com distribuição global, causando epidemias sazonais. Os sinais e sintomas vão desde formas assintomáticas até formas graves com complicações tais como: pneumonia bacteriana, sinusite, otite, desidratação, piora de doenças crônicas como insuficiência cardíaca, asma ou diabetes (Brasil, 2017). No cenário atual, os resultados de um estudo revelaram que um maior número de sintomas gripais em geral possa levar ao diagnóstico de gripe ou resfriado comum (Czubaket al, 2021). Para uma prevenção efetiva desse agravo é necessária vigilância qualificada e vacinação (Brasil, 2017), que é considerada uma das intervenções mais custo-efetivo e segura (Make, 2015).

Os dados atuais da Organização Mundial da Saúde (OMS) apontam que houve um aumento substancial na expectativa de vida da população mundial entre os anos 2000 a 2015, apresentando uma média de 71,4 anos, considerando mulheres e homens (Alves, et al, 2017). O que reforça a necessidade da intensificação das políticas de saúde para o envelhecimento saudável da população.

Dentre essas políticas, temos a vacinação demonstrando uma relação positiva na prevenção de formas graves da influenza. Estudos demonstram os benefícios da vacinação entre idosos e portadores de doenças crônicas, como redução dos casos de pneumonias e de óbitos por complicações decorrentes da influenza (Bekkat-Berkani, et al, 2017).

Entretanto, é necessária uma maior divulgação e recomendação da vacinação para os segmentos populacionais mais reticentes à absorção de vacinas, principalmente a população idosa, aumentando as taxas de coberturas vacinais (Sato, et al, 2015a). Na França, no período de vacinação de outubro de 2016 a janeiro de 2017, apenas 63,5\% dos idosos foram vacinados, e o que mais colaborou para a baixa cobertura foi a falta de recomendação da vacinação por profissionais da saúde (Casalino, et al, 2018).

Vários fatores relacionados à população idosa influenciam as coberturas vacinais da influenza como fatores sociodemográficos, familiares e individuais (Oliveira, et al, 2014) além da situação conjugal, prática de atividade física e interação com o serviço de saúde serem considerados como fatores que podem influenciar na adesão à vacinação (Sato, et al, 2020). A escolaridade é citada como fator determinante para vacinação. O baixo nível de escolaridade reflete diretamente na condição de saúde, pois muitas informações podem deixar de ser compreendidas, inclusive aquelas relativas à imunização, podendo gerar uma não adesão à vacinação (Oliveira, et al, 2014). 
Research, Society and Development, v. 10, n. 6, e3610615222, 2021

(CC BY 4.0) | ISSN 2525-3409 | DOI: http://dx.doi.org/10.33448/rsd-v10i6.15222

Segundo Rodrigues et al, os idosos deixam de vacinar por motivos religiosos, filosóficos e também pela desinformação quanto aos riscos de eventos adversos. Tais motivos também foram encontrados em um estudo realizado no EUA onde os idosos deixaram de vacinar por medo dos efeitos colaterais, preocupação com a eficácia da vacina e por acreditarem que a vacina não é necessária. A desinformação e experiências negativas anteriores com a vacina também tiveram contribuição na redução coberturas vacinais (Rodrigues,et al, 2014).

Investigação realizada na Austrália demonstrou que a cobertura vacinal aumenta de acordo com o aumento da idade. Os idosos na faixa etária de 60-64 anos apresentam aderência de apenas 34,5\%, essa porcentagem aumenta gradativamente e atinge seu pico na faixa etária de $80-84$ anos com 70,4\%. Outros fatores que influenciaram para a vacinação foram: sexo feminino, não ter ensino superior e possuir renda familiar anual $<\mathrm{ou}=\$ 50,000$ (Dyda, et al, 2015). Dentre os motivos de não adesão a vacinação na Polônia foi identificada a falta de eficácia da vacina, a falta de tempo, o alto custo e o complicado procedimento de vacinação no país (Ganczak, et al, 2017).

Na Suíça um estudo aponta a diminuição da cobertura vacinal em idosos entre 65-75 anos de idade comparado aos últimos anos entre 2012 a 2017, demonstrando fatores que podem influenciar diretamente a adesão de idosos a vacinação contra Influenza, sendo eles: idosos que vivem em áreas urbanas; história de tabagismo; má auto-reportagem do estado de saúde; possuir seguro saúde; ter uma profissão médica; ser portador de doença crônica (Zürcher, et al, 2021).

Já no Brasil, os resultados de um estudo evidenciam as coberturas vacinais contra influenza em idosos tem se mantido acima da média esperada desde o ano de 2011, aumentando significativamente nos últimos anos, obtendo valores próximos ou superiores a 100\% de cobertura em 2019 (Azambuja, et al, 2020).

Um estudo realizado com objetivo de identificar a cobertura vacinal contra influenza em idosos cadastrados em uma unidade de saúde da família no município de Montes Claros-MG identificou que o fato de ter menos de 80 anos, ser casado/união estável, e ter renda menor de 2 salários mínimos influenciou positivamente na adesão à vacinação (Santoset al, 2014). Outro aspecto constatado foi à relação da escolaridade com a aceitação da vacina. Dentre os idosos que receberam a vacina a maioria deles (62\%) era analfabeta, enquanto na parcela dos não vacinados a maioria deles $(62,5 \%)$ era alfabetizada, podemos concluir então que neste caso, a escolaridade serviu como um fator negativo a adesão a vacinação o que contradiz as expectativas do Ministério da Saúde (MS) que espera que a população com maior grau de instrução apresente maior taxa de vacinação (Santos, et al, 2014).

Já no município de Petrolina-PE um estudo analisou os motivos de adesão e não adesão a vacinação e identificou que o sexo, a idade e a inserção no mercado de trabalho estiveram relacionados. Foi possível identificar que a maioria dos idosos vacinados eram mulheres de idade média de 70,3 anos e aposentadas, já os idosos não vacinados eram na sua maioria homens trabalhadores com idade média de 66,4 anos (Silva, et al, 2013).

Em São Paulo o estudo de Sato et al. demonstrou em seus resultados a associação da adesão a vacinação pelos idosos à sua situação conjugal sendo que, idosos sem companheiro (a) apresentaram menor cobertura vacinal quando relacionados aos que possuem. Além disso, o estudo demonstrou ausência da diferença das coberturas vacinais contra Influenza entre os variados estratos de escolaridade (Sato, et al, 2020).

Estudo realizado em São Paulo corrobora outros estudos apontando que idosos com a idade mais avançada apresentam predisposição à vacinação. O estudo também demostrou que a absorção da vacina não estava relacionada ao status socioeconômico, o que também é um achado relevante (Sato, 2015b).

Além das características relacionadas aos idosos outros fatores, como a organização da atenção primária a saúde, podem comprometer a cobertura vacinal. Segundo Braz et al. a estratégia saúde da família impacta positivamente nas ações de imunoprevenção (Braz, al, 2016). Entretanto, em uma investigação realizada em Minas Gerais, em 2013, com o objetivo de 
Research, Society and Development, v. 10, n. 6, e3610615222, 2021

(CC BY 4.0) | ISSN 2525-3409 | DOI: http://dx.doi.org/10.33448/rsd-v10i6.15222

comparar indicadores de uso e qualidade dos serviços de saúde entre residentes em área coberta pela ESF identificou que a cobertura vacinal de influenza não sofreu mudança significativa em áreas cobertas pela ESF (Lima-Costa, Turci \& Macinko, 2013).

Temos a vacinação como uma ferramenta para proporcionar um envelhecimento saudável. Por meio da cobertura vacinal temos conhecimento da adesão vacinal, que sofre influência de diversos fatores. Se faz necessário conhecê-los e analisálos para propor estratégias a fim de captar um maior número de idosos, aumentar a cobertura vacinal e por fim contribuir para senescência.

Tendo em vista a importância da vacinação para o idoso, este estudo objetivou analisar a cobertura vacinal antiinfluenza em idosos em Minas Gerais (MG).

\section{Método}

Trata-se de um estudo transversal, realizado na região Oeste de MG entre abril de 2018 e julho de 2019, utilizando os dados da campanha de vacinação contra influenza em 2017.

Por meio do estudo transversal é possível determinar de forma simultânea a exposição e a condição de saúde dos participantes possibilitando ao pesquisador a análise da existência de associação entre a exposição do indivíduo e uma doença ou condição sendo que, ambos eventos estão relacionados à saúde, permitindo que sejam realizadas inferências de associações no estudo realizado (Lima-Costa \& Barreto, 2003; Silva et al. 2014).

A região Oeste localiza-se entre as regiões Central, Sul e Alto Paranaíba do estado de MG. Possui vasta extensão territorial, com $31.543 \mathrm{~km}^{2}$, sendo composta por 53 municípios (IBGE, 2010). Ela conta com seis regiões de saúde, considerandose a base territorial de planejamento da atenção à saúde, que são: Bom Despacho, Campo Belo/Santo Antônio do Amparo, Divinópolis/Santo Antônio do Monte, Formiga, Itaúna e Pará de Minas. Foram incluídos neste estudo todos os 53 municípios da região que participaram da campanha vacinal de influenza de 2017 e tinham os dados digitados no Sistema de Informação do Programa Nacional de Imunização (SIPNI).

Os dados foram coletados das bases do SIPNI, Sistema de Informação da Atenção Básica - SISAB e Instituto Brasileiro de Geografia e Estatística - IBGE.

Foram analisadas as variáveis: cobertura vacinal, porte populacional e cobertura de estratégia de saúde da família (ESF). A variável desfecho foi cobertura vacinal anti-influenza em pessoas com 60 anos e mais e as variáveis de exposição o porte populacional e a cobertura de ESF. Procurou-se analisar na região, a cobertura vacinal anti-influenza em idosos e se essa cobertura foi influenciada pela cobertura de ESF e pelo porte populacional dos municípios.

A cobertura vacinal mínima anti-influenza foi definida em $90 \%$ para a população idosa, conforme norma estabelecida pelo MS (Brasil, 2017). Os municípios foram estratificados entre aqueles que possuíam cobertura vacinal menor que $90 \%$ e os municípios que possuíam cobertura vacinal maior ou igual a $90 \%$.

Em relação a cobertura de ESF, os municípios foram estratificados entre aqueles com cobertura igual ou menor de $80 \%$ e os municípios com cobertura maior de 80\%. A estratificação seguiu a recomendação do Departamento de Atenção Básica (DAB) (Brasil, 2008). Quanto ao porte populacional os municípios foram classificados em menores de 10.000 habitantes, de 10.000 a 50.000 habitantes e maior de 50.000 habitantes. Esta estratificação foi baseada no referencial do PQAVS (Programa de Qualificação das Ações de Vigilância em Saúde) (Brasil, 2013).

Inicialmente foi realizada a análise descritiva das variáveis estudadas e calculada a cobertura vacinal de cada município. Para determinar a associação entre a cobertura vacinal e as das variáveis de exposição foi utilizado o teste de qui-quadrado de 
Research, Society and Development, v. 10, n. 6, e3610615222, 2021

(CC BY 4.0) | ISSN 2525-3409 | DOI: http://dx.doi.org/10.33448/rsd-v10i6.15222

Pearson fixando um nível de significância de 5\%, e para estimar a força de associação razão de chances (oddsratio) por meio do teste de Mantel-Haenszel com seus respectivos intervalos de confiança de 95\%. Para análise dos dados, utilizou-se o software Statístical Pocckage for the Social Sciences (SPSS®) for Windows versão 17.0.

Este projeto foi aproado no CEPES da Universidade Federal de São João Del Rei (UFSJ), parecer nº 009522/2018 e CAAE no 83082918.7.0000.5545.

\section{Resultados}

A população da Região Oeste de MG é constituída por 1.172.707 habitantes, o que corresponde a aproximadamente $6 \%$ do total do Estado. Dentre esses, 12,1\% são idosos, o que corresponde a 142.547 habitantes.

Em relação ao total de idosos, a região de Santo Antônio do Monte/Divinópolis apresentou a maior população de idosos (35,6\%), seguida pelas regiões de Santo Antônio do Amparo/ Campo Belo (19,4\%), Pará de Minas (12,9\%), Formiga (12,6\%), Itaúna $(9,8 \%)$ e Bom Despacho $(9,7 \%)$. Quando analisamos os dados proporcionalmente por região, a que apresenta a maior proporção de idosos é a região de Santo Antônio do Amparo/Campo Belo (14,2\%), seguida pelas regiões de Formiga e Bom Despacho (ambas com aproximadamente 13,8\%), Itaúna (12,1\%), Santo Antônio do Monte/Divinópolis (11,8\%) e Pará de Minas $(9 \%)$.

Em relação ao porte populacional dos municípios, identificou-se que a maioria tem até 50.000 habitantes $(88,7 \%)$ (Tabela 1).

Tabela 1. Porte populacional dos municípios da Região Ampliada Oeste-MG, no ano de 2017.

\begin{tabular}{|c|c|c|}
\hline \multirow{2}{*}{ Porte populacional } & \multicolumn{2}{|c|}{ Número de municípios } \\
\hline & $\mathbf{N}$ & $\%$ \\
\hline Até 10.000 habitantes & 27 & 46,2 \\
\hline De 10.001 a 50.000 habitantes & 20 & 39,0 \\
\hline Acima de 50.001 habitantes & 6 & 14,8 \\
\hline Total & 53 & 100 \\
\hline
\end{tabular}

Fonte: Instituto Brasileiro de Geografia e Estatística - IBGE

A região Oeste possui 323 estratégias saúde da família (ESF), o que garante uma cobertura média de 94,7\%. A região de saúde que apresentou a maior cobertura de ESF foi a de Santo Antônio do Amparo/Campo Belo (99,3\%), seguido pelas regiões de Bom Despacho e Formiga (ambas com 98,4\%), Itaúna (94,6\%), Pará de Minas (89,8\%) e Divinópolis/Santo Antônio do Monte $(88,1 \%)$. (Tabela 2).

A cobertura vacinal anti-influenza em idosos na Região Oeste foi de 95,6\%. Entre os 53 municípios desta região, a maioria (84,9\%) alcançou a meta de cobertura vacinal de influenza de 90\%, conforme preconiza o MS (Brasil, 2017). A média total de cobertura vacinal da Região Oeste foi de 95,2\%, sendo a região de saúde de Bom Despacho a que apresentou maior cobertura vacinal $(98,8 \%)$. Ao analisar a cobertura por municípios, verificamos que sete deles não atingiram a cobertura de $90 \%$ e, por outro lado, oito deles atingiram cobertura vacinal de $100 \%$. 
Tabela 2. Cobertura de Estratégia Saúde da Família e cobertura vacinal contra influenza, por região de saúde, Região Oeste de Minas Gerais, 2017.

\begin{tabular}{|c|c|c|c|c|}
\hline Região de Saúde & $\begin{array}{c}\text { População idosa } \\
\text { (n) }\end{array}$ & $\begin{array}{c}\mathbf{E S F}^{1} \\
(\mathbf{n})\end{array}$ & $\begin{array}{c}\text { Cobertura } \text { ESF }^{1} \\
(\%)\end{array}$ & $\begin{array}{c}\text { Cobertura Vacinal } \\
(\%)\end{array}$ \\
\hline Santo Antônio do Amparo/ Campo Belo & 27.631 & 67 & 99,3 & 94,2 \\
\hline Divinópolis/ Santo Antônio do Monte & 50.756 & 96 & 88,1 & 96 \\
\hline Bom Despacho & 13.896 & 34 & 98,4 & 98,8 \\
\hline Pará de Minas & 18.370 & 56 & 91,2 & 93,8 \\
\hline Formiga & 17.969 & 40 & 98,4 & 92,6 \\
\hline Itaúna & 13.925 & 33 & 94,6 & 98 \\
\hline Região Ampliada Oeste- MG & 142.547 & 326 & 95 & 95,6 \\
\hline
\end{tabular}

${ }^{1}$ ESF: Estratégia Saúde da Família. Fonte: SIPNI, Sistema de Informação da Atenção Básica - SISAB.

As variáveis porte populacional e cobertura de ESF não estiveram associadas à cobertura vacinal, apresentando um $\mathrm{p}$ valor de 0,697 e 0,949, respectivamente (Tabela 3).

Tabela 3. Cobertura vacinal contra a influenza segundo as variáveis porte populacional dos municípios e cobertura de Estratégia Saúde da Família (ESF), Região Oeste, 2017.

\begin{tabular}{|c|c|c|c|}
\hline Variáveis & & a Vacinal & pvalor $^{1}$ \\
\hline Porte populacional & $<90 \%$ & $90 \%$ e mais & \multirow{4}{*}{0,697} \\
\hline Até 10.000 habitantes & $11,1 \%$ & 88,9 & \\
\hline De 10.001 à 50.000 habitantes & $20 \%$ & 80,0 & \\
\hline Acima de 50.001 habitantes & $16,7 \%$ & 83,3 & \\
\hline \multicolumn{4}{|l|}{ Cobertura ESF ${ }^{1}$} \\
\hline Menor de $80 \%$ & $14,3 \%$ & $85,7 \%$ & \multirow{2}{*}{0,949} \\
\hline $80 \%$ e mais & $15,2 \%$ & $84,8 \%$ & \\
\hline
\end{tabular}

${ }^{1} \mathrm{p}$ valor: Probabilidade de significância- Teste de qui-quadrado de Pearson. Fonte: SIPNI, Sistema de Informação da Atenção Básica SISAB.

\section{Discussão}

O presente estudo não apresentou associação entre a presença da ESF e a cobertura vacinal, sendo possível demonstrar a cobertura vacinal em todas as regiões de saúde da região oeste de Minas Gerais, o que leva a crer que a vacinação contra influenza já está consolidada e se tornou algo rotineiro para os idosos. Pressupõe-se que os idosos reconhecem os benefícios da vacina contra a influenza e que isso se deve às práticas promocionais realizadas pelos serviços. Fato esse que pode não ser evidenciado pelos números, mas a consolidação dessa campanha provavelmente muito se deve ao papel que vem sido exercido por esse modelo de assistência.

Uma das efetivações do compromisso do governo brasileiro com a universalidade, a integralidade e a equidade da atenção à saúde na área de imunizações é a campanha nacional de vacinação contra a gripe, que é destinada a população de 60 
Research, Society and Development, v. 10, n. 6, e3610615222, 2021

(CC BY 4.0) | ISSN 2525-3409 | DOI: http://dx.doi.org/10.33448/rsd-v10i6.15222

anos ou mais, bem como para as crianças de seis meses a menores de cinco anos; professores; trabalhadores da saúde (tanto da rede pública como particular); povos indígenas; gestantes; puérperas (mulheres até 45 dias após o parto); população privada de liberdade; funcionários do sistema prisional e portadores de doenças crônicas não transmissíveis ou com outras condições clínicas especiais (respiratórias, cardíacas, renais, hepáticas, neurológicas, diabéticos, obesos, imunossuprimidos e transplantados), beneficiando assim os que mais necessitam da vacina, atendendo deste modo, aos princípios fundamentais do Sistema Único de Saúde (Brasil, 2017).

Logo, a vacinação contra influenza está associada a uma diminuição média de $27 \%$ no risco de hospitalização por pneumonia ou influenza e de $48 \%$ no risco de morte por qualquer causa (Bós, et al, 2013).

Apesar de a cobertura vacinal da influenza, na região de saúde, atingir a meta de $90 \%$, é necessário a discutir sobre a heterogeneidade de coberturas vacinais, tendo em vista, que 15,1\% dos municípios não atingiram a meta mínima preconizada pelo MS. A heterogeneidade nas coberturas vacinais leva ao risco de acúmulo de indivíduos susceptíveis ao agente infeccioso, influenciando assim no processo de imunização. Cabe ao enfermeiro ter conhecimento dessas variações a fim de realizar uma busca ativa para detectar qual o motivo pelo qual a meta não está sendo alcançada e propor medidas resolutivas (Nora, et al, 2016).

Muitos estudos trazem os benefícios da ESF em indicadores de saúde como diminuição da mortalidade infantil, menores taxas de internações hospitalares e também impacto na disponibilidade de vacinas. Estudos comparativos entre os modelos de atenção primária são escassos na literatura, principalmente em relação ao impacto gerado com a imunização, o que impede conclusões mais apropriadas (Araújo, et al, 2017).

Esse modelo de atenção primária a saúde permitiu uma mudança significativa no conceito de saúde e cuidado, com a inserção de uma equipe multidisciplinar e a inversão do modelo biomédico e curativista. O paciente é visto de maneira integral e as ações de promoção a saúde e prevenção de doenças, como prática de exercícios físicos, alimentação saudável, imunização dentre outras, tornam-se mais evidentes (Sorato, et al, 2015). Além disso, a forma de organização da ESF permite a criação de um vínculo maior com o paciente, contribuindo para mudanças de comportamento, adesão a tratamentos e hábitos saudáveis (Silva, et al, 2015).

Uma investigação realizada no Rio Grande do Sul identificou que os municípios que atingiram a meta de cobertura vacinal de $80 \%$ preconizada em 2016 obtiveram taxa significativamente menores de mortes por doenças respiratórias (Bós, et al, 2013). Portanto é comprovado o impacto positivo causado pela vacina na saúde dos idosos, o que torna a campanha extremamente necessária para o controle da Influenza.

Visando melhorar a vigilância em saúde, novas tecnologias foram sendo incorporadas nos serviços de saúde, como a utilização dos Sistemas de Informação em Saúde (SIS) para a tomada de decisões (Silva, et al, 2015). O SIPNI contribui para que a população alvo das campanhas seja detectada com maior facilidade e acionada para adesão a vacinação (Silva, et al, 2018), além de fornecer maiores informações sobre o usuário vacinado, avaliar as coberturas vacinais e, consequentemente, auxiliar no norteamento das ações de imunização (Sato, 2015b). Estudo realizado na Bahia, com secretários de saúde, mostra que ainda falta qualificação dos profissionais para utilizarem o sistema, consequentemente, a ferramenta apesar de oferecer os recursos necessários para uma boa coleta de dados, não é utilizada por completo (Pinheiro, et al, 2016).

Por este motivo, o presente estudo teve como uma de suas limitações à incompletude dos dados no SIPNI, inviabilizando a avaliação do perfil sóciodemográfico e de outras variáveis relacionadas à vacinação do idoso. 
Research, Society and Development, v. 10, n. 6, e3610615222, 2021

(CC BY 4.0) | ISSN 2525-3409 | DOI: http://dx.doi.org/10.33448/rsd-v10i6.15222

\section{Considerações Finais}

A Região Oeste de Minas Gerais possui alta cobertura vacinal anti-influenza em idosos e essa cobertura não foi influenciada pela ESF ou porte populacional dos municípios. Este estudo se faz relevante por demonstrar dados importantes relacionados à cobertura vacinal anti-influenza em idosos na região de saúde Oeste do estado de Minas Gerais (MG).

Como limitações deste estudo destaca-se a qualidade da informação do SIPNI, que apesar de fornecer informações relacionadas às características individuais dos idosos, neste estudo tais informações não puderam ser avaliadas devido à incompletude do banco de dados avaliado.

Devido à heterogeneidade das coberturas vacinais entre os idosos, é necessário que as unidades de saúde da atenção básica realizem uma busca ativa desta população rotineiramente, com o intuito de descobrir qual o motivo da baixa cobertura vacinal visto que foram encontrados poucos estudos relacionados aos motivos da não adesão a vacinação pela população estudada.

É necessário a realização de estudos atuais que aprofundem mais no que se diz respeito à vacinação e cobertura vacinal anti-influenza em idosos. Novos estudos, portanto, poderão contribuir com a formulação de políticas públicas mais adequadas que enalteçam os benefícios decorrentes da imunização de idosos, com o objetivo de estimular à adesão a vacinação contra Influenza, aumentando assim a cobertura vacinal entre esta faixa etária.

Além disso, é preciso que haja um incentivo para utilização correta do sistema, realizado através de capacitações que abranjam toda equipe da rede de assistência, contribuindo assim para gestão do serviço com dados palpáveis e fidedignos que demonstrem dados próximos a realidade da população favorecendo assim a produção de conhecimento científico.

\section{Referências}

Alves, B. L. (2017). Hospital de maternidade nova vida: o uso da medicina preventiva como modelo de gestão para a área da saúde. Revista Inovação $e$ Tecnologia, 1(1), 35-42.

Araújo, W. R. M., Queiroz, R. C. D. S., Rocha, T. A. H., Silva, N. C. D., Thumé, E., Tomasi, E., ... \& Thomaz, E. B. A. F. (2017). Estrutura e processo de trabalho na atenção primária e internações por condições sensíveis. Revista de Saúde Pública, 51, 75.

Azambuja, H. C. S., Carrijo, M. F., Martins, T. C. R., \& Luchesi, B. M. (2020). O impacto da vacinação contra influenza na morbimortalidade dos idosos nas regiões do Brasil entre 2010 e 2019. Cadernos de Saúde Pública, 36, e00040120.

Bekkat-Berkani, R., Wilkinson, T., Buchy, P., Santos, G., Stefanidis, D., \& Meyer, N. (2017). Seasonal influenza vaccination in patients with COPD: a systematic literature review. BMC Pulmonary Medicine 17:79.

Bós, Â. J. G., \& Mirandola, A. R. (2013). Cobertura vacinal está relacionada à menor mortalidade por doenças respiratórias. Ciência \& Saúde Coletiva, 18(5), 1459-1462.

Brasil. Ministério da Saúde. (2008). Departamento de Atenção Básica. Evolução e série histórica de implantação da ESF: relatório de busca.

Brasil. Ministério da Saúde. (2013). Gabinete do Ministro. Portaria no 1708, de 16 de março de 2013. Regulamenta o Programa de Qualificação das Ações de Vigilância em Saúde (PQAVS), com a definição de suas diretrizes, financiamento, metodologia de adesão e critérios de avaliação dos Estados, Distrito Federal e Municípios. Diário Oficial da República Federativa do Brasil, nov 2013; Seção 1.

Brasil. Ministério da Saúde. (2017). Informe Técnico. 19ª Campanha Nacional de Vacinação contra a Influenza.

Braz, R. M., Domingues, C. M. A. S., Teixeira, A. M. D. S., \& Luna, E. J. D. A. (2016). Classificação de risco de transmissão de doenças imunopreveníveis a partir de indicadores de coberturas vacinais nos municípios brasileiros. Epidemiologia e serviços de saúde, 25, $745-754$.

Casalino, E., Ghazali, A., Bouzid, D., Antoniol, S., Pereira, L., Kenway, P., ... \& Emergency Department study group on respiratory viruses. (2018). Patient's behaviors and missed opportunities for vaccination against seasonal epidemic influenza and evaluation of their impact on patient's influenza vaccine uptake. Plos one, 13(3), e0193029.

Czubak, J., Stolarczyk, K., Orzeł, A., Frączek, M., \& Zatoński, T. (2021). Comparison of the clinical differences between COVID-19, SARS, influenza, and the common cold: A systematic literature review. Advances in Clinical and Experimental Medicine, 30(1), 109-114.

Da Silva, D., Lopes, E. L., \& Junior, S. S. B. (2014). Pesquisa quantitativa: elementos, paradigmas e definições. Revista de Gestão e Secretariado, 5(1), 01-18. 
Research, Society and Development, v. 10, n. 6, e3610615222, 2021

(CC BY 4.0) | ISSN 2525-3409 | DOI: http://dx.doi.org/10.33448/rsd-v10i6.15222

Dyda, A., MacIntyre, C. R., McIntyre, P., Newall, A. T., Banks, E., Kaldor, J., \& Liu, B. (2015). Factors associated with influenza vaccination in middle and older aged Australian adults according to eligibility for the national vaccination program. Vaccine, 33(29), 3299-3305.

Ganczak, M., Gil, K., Korzeń, M., Bażydło, M. (2017). Cobertura e Determinantes da Influenza da Vacinação contra Influenza em Pacientes Idosos em um País com Baixa Implementação de Vacinação. International Journal Environmental Reseach and Publich Health [Internet];14(6):665.

IBGE. Instituto Brasileiro de Geografia e Estatística [homepage na internet]. Contagem da população de 2010.

Lima-Costa, M. F., \& Barreto, S. M. (2003). Tipos de estudos epidemiológicos: conceitos básicos e aplicações na área do envelhecimento. Epidemiologia e serviços de saúde, 12(4), 189-201.

Lima-Costa, M. F., Turci, M. A. \& Macinko, J. (2013). Estratégia Saúde da Família em comparação a outras fontes de atenção: indicadores de uso e qualidade dos serviços de saúde em Belo Horizonte, Minas Gerais, Brasil. Cadernos de Saúde Pública, 29(7), 1370-1380.

Make, S. (2015). Vaccine coverage a key un health indicator. Nature [Internet]; 523:165.

Nora, T. T. D., Paz, A. A., Linch, G. F. D. C., Pelegrini, A. H. W., \& Wachter, M. Z. D. (2016). Situação da cobertura vacinal de imunobiológicos no período de 2009-2014. Revista Enfermagem UFSM, 482-493.

Oliveira, M. F. S. D., Martinez, E. Z., \& Rocha, J. S. Y. (2014). Fatores associados à cobertura vacinal em menores de cinco anos em Angola. Revista de Saúde Pública, 48, 906-915.

Pereira, A. S., Shitsuka, D. M., Parreira, F. B., \& Shitsuka, R. (2018). Metodologia da pesquisa científica [recurso eletrônico. UFSM.

Pinheiro, A. L. S., Andrade, K. T. S., de Oliveira Silva, D., Zacharias, F. C. M., Gomide, M. F. S., \& Pinto, I. C. (2016). Gestão da saúde: o uso dos sistemas de informação e o compartilhamento de conhecimento para a tomada de decisão. Texto \& Contexto Enfermagem, 25(3), 1-9.

Rodrigues, C. L., Kobiraki, C. M., Gonçalves, E. M. S., Lúcio, L. M., Iannarelli, M. D. J. B. M., \& Lodovici, F. M. M. (2014). A relevância da Imunização para a Longevidade e a necessária anuência de indivíduos do "Programa Acompanhante de Idosos". Revista Kairós: Gerontologia, 17(4), $31-48$.

Sato, A. P. S., Antunes, J. L. F., Moura, R. F., de Andrade, F. B., Duarte, Y. A. O., \& Lebrão, M. L. (2015). Factors associated to vaccination against influenza among elderly in a large Brazilian metropolis. PloS one, 10(4), e0123840.

Sato, A. P. S. (2015b). Programa Nacional de Imunização: Sistema Informatizado como opção a novos desafios. Revista de Saúde Pública, 49 , 39.

Sato, A. P. S., Andrade, F. B. D., Duarte, Y. A. O., \& Antunes, J. L. F. (2020). Cobertura vacinal e fatores associados à vacinação contra influenza em pessoas idosas do Município de São Paulo, Estudo SABE 2015. Cadernos de Saúde Pública, 36, e00237419.

Silva, B. S., Coelho, H. V., Cavalcante, R. B., Oliveira, V. C. D., \& Guimarães, E. A. A. (2018). Evaluation study of the national immunization program information system. Revista brasileira de enfermagem, 71, 615-624.

Silva, L.B. (2018). Sistemas de informações em saúde como ferramenta para gestão do SUS. Caderno Saúde e Desenvolvimento. 7:52-62.

Silva, S. P. C., \& Menandro, M. C. S. (2013). Representações de idosos sobre a vacina da gripe. Ciência \& Saúde Coletiva, 18, $2179-2188$.

Soratto, J., Pires, D. E. P. D., Dornelles, S., \& Lorenzetti, J. (2015). Estrategia de Salud de la Familia: una innovación tecnológica en salud. Texto \& ContextoEnfermagem, 24(2), 584-592.

Souza Santos, C. L., dos Reis Alves, C., dos Reis Alves, M., Teles, M. A. B., da Silva, D. M., \& da Fonseca, J. L. T. (2014). Vacinação contra influenza em idosos residentes na comunidade. Revista de Pesquisa Cuidado é Fundamental Online, 6(3), 876-881.

Zürcher, K., Zwahlen, M., Berlin, C., Egger, M., \& Fenner, L. (2021). Losing ground at the wrong time: trends in self-reported influenza vaccination uptake in Switzerland, Swiss Health Survey 2007-2017. BMJ open, 11(2), e041354. 